\title{
Editorial: ICE and Small
}

\author{
Adam P. Roberts \\ Department of Parasitology, Liverpool School of \\ Tropical Medicine, Pembroke Place, Liverpool, \\ L3 5QA, UK
}

Correspondence: Adam.Roberts@lstmed.ac.uk

DOI: https://dx.doi.org/10.21775/cimb.029.001

Bacterial genomes vary considerably in terms of size and gene content. The proportion of a genome composed of horizontally acquired DNA or mobile genetic elements also varies, but follows an ecological pattern with more mobile genetic element genes being found in facultative intracellular bacteria than those considered extracellular and both containing more than obligately intracellular bacteria (Newton and Bordenstein; 2011). As bacterial genomes evolve they gain and lose genes (and DNA) with extreme examples of genome reduction being seen in bacterial endosymbionts such as Wolbachia spp. As the relationship between the two selective units (endosymbiont and host) becomes more intertwined and the distinction between intracellular independent bacterial symbiont and organelle blurs, the benefits of genetic variation reduce and the genome inevitably becomes smaller.

However, just because the genome of a host is small it does not translate to a lack of opportunity for mobile DNA to exploit. Wolbachia spp. for example, have insertion sequences and prophages. In this issue of CIMB there are two detailed reviews of horizontal gene transfer mechanisms used by members of phylogenetic families of bacteria, which are characterised by having relatively small genomes. One (Citti et al.,2018) describes pervasive horizontal gene transfer in genera of the Mollicutes family (Mycoplasma, Ureoplasma and Spiroplasma) whilst the other (Blesa et al., 2018) reviews horizontal gene transfer in Thermus spp., a genus within the Thermaceae family.
Central to the movement of DNA amongst these bacteria are integrative and conjugative elements, or ICEs. Usually ICEs exist in an integrated form within their host bacterial genome. These mobile genetic elements can detect environmental cues triggering their excision, transfer and reintegration into a host replicon (Johnson and Grossman, 2015). Importantly they also usually carry cargo genes encoding accessory functions not involved with their mobility. These accessory functions e.g. antimicrobial resistance, often provide the selective advantage to the host in certain environments which enable ICEs to spread throughout bacterial populations as well as the spread of their bacterial hosts within specific niches. It is also likely that ICEs act as recombination substrates for other similar ICEs which enter the same cell.

Once thought to be utterly dependant on their host for replication and solely involved in conjugation of themselves between donor and recipient cells, the work described in the reviews in this issue of CIMB underlines the growing appreciation of the complex life-styles of ICEs and the interdependent associations between different mechanisms of horizontal gene transfer, and other families of mobile genetic elements, which facilitate ICE movement from one cell to another. What is increasingly evident, and may be particularly important if you are a bacterium with a small genome, is that ICEs can catalyse the transfer of more than just self; potentially mobilising large sections of the host genome and facilitating genetic recombination and rearrangements. As evolution usually selects for efficient solutions to problems it is worth contemplating whether the presence of ICEs in small genomes is perhaps the best way to avoid the effects of Muller's ratchet (Muller, 1964).

\section{References}

Blesa A, Averhoff B and Berenguer J. (2018). Horizontal gene transfer in Thermus spp. Curr. 
Issues Mol. Biol. 29: 23-36. https://dx.doi.org/ 10.21775/cimb.029.023

Citti C, Dordet-Frisoni E, Nouvel LX, Kuo CH and Baranowski E. (2018). Horizontal gene transfers in mycoplasmas (mollicutes) Curr. Issues Mol. Biol. 29: 3-22. https://dx.doi.org/10.21775/cimb. 029.003

Johnson CM andGrossman AD. (2015). Integrative and Conjugative Elements (ICEs): What they do and how they work. Annu Rev Genet. 49: 577-601. doi: 10.1146/annurev-genet-112414-055018.
Muller HJ (1964). The relation of recombination to mutational advance. Mutat Res. 106: 2-9. PMID 14195748.

Newton IL and Bordenstein SR. (2011). Correlations between bacterial ecology and mobile DNA.

Curr Microbiol. 62:198-208. https://dx.doi.org/ $10.1007 / \mathrm{s} 00284-010-9693-3$. 